http://dx.doi.org/10.35381/e.k.v4i1.1462

\title{
Aprendizaje Invertido en el desarrollo de habilidades de escucha en la asignatura de Inglés
}

Aprendizaje Invertido en el development de habilidades de escucha en la assignature

\author{
Tatiana Carolina Trelles-Campuzano \\ tatiana.trelles@est.ucacue.edu.ec \\ Universidad Católica de Cuenca, Azogues \\ Ecuador \\ https://orcid.org/0000-0002-6116-7096 \\ Ana Zulema Castro-Salazar \\ azcastros@ucacue.edu.ec \\ Universidad Católica de Cuenca, Azogues \\ Ecuador \\ https://orcid.org/0000-0002-3837-314X
}

Recepción: 10 de agosto 2021

Revisado: 15 de septiembre 2021

Aprobación: 15 de noviembre 2021

Publicación: 01 de diciembre 2021 


\title{
RESUMEN
}

La siguiente investigación trata el problema de la dificultad que tienen los estudiantes de tercero bachillerato para entender y escuchar el idioma Inglés en la adquisición como segunda lengua. En tal sentido el objetivo es analizar la estrategia metodológica aula invertida para el progreso de la habilidad escucha dentro de las clases de Inglés. Esta investigación conduce al desarrollo de las estrategias didácticas en los docentes y permite analizar cuán importante es el uso de las metodologías activas en la actualidad. De metodología descriptiva. El aprendizaje Invertido como estrategia didáctica para el desarrollo de habilidades de escucha en la asignatura de Inglés es una gran opción para ser puesta en manifiesto en el aula de clases, puesto que permite la participación activa de los estudiantes no solo en la destreza de escuchar, sino en las de hablar y escribir.

Descriptores: Aprendizaje verbal; método de aprendizaje; estudio en casa. (Palabras tomadas del Tesauro UNESCO).

\begin{abstract}
The following research deals with the problem of the difficulty that third year high school students have to understand and listen to the English language when acquiring a second language. In this sense, the objective is to analyze the inverted classroom methodological strategy for the progress of listening skills within English classes. This research leads to the development of teaching strategies in teachers and allows us to analyze how important the use of active methodologies is today. Descriptive methodology. Inverted learning as a didactic strategy for the development of listening skills in the English subject is a great option to be shown in the classroom, since it allows the active participation of students not only in the skill of listening, but in speaking and writing.
\end{abstract}

Descriptors: Verbal learning; learning methods; home study. (Words taken from the UNESCO Thesaurus). 


\section{INTRODUCCIÓN}

La Educacion pública segun (Hernández-Hernández \& Isquierdo-Sandoval, 2020), mencionan los cambios educativos que han tenido que realizar con respecto a la enseñanza del idioma ingles como lengua extranjera, dentro de las dimensiones que menciona es la practica pedagogica haciendo referencia a la carencia de usos metodologicos dentro de las clases. En el Ecuador el Inglés es definido como idioma en lengua extranjera, dado que no es propia o nativa del país, en este sentido el idioma Inglés se lo emplea como vital importancia para diferentes fines.

Lo que representa un gran desafío para los docentes el enseñar esta materia en el empleo de las metodologías y en la motivación para aprenderlo sobre todo si aún el nivel de preparación de los profesores es bajo. Por consiguiente, el (Ministerio de Educación [MINEDUD] 2014), luego de una evaluación a los docentes con resultados no tan favorable ha creado varios programas de fortalecimiento para el aprendizaje del Inglés. El Inglés desde un punto de vista adquirido como lengua extranjera en sus inicios fue considerado tan solo una materia optativa, es decir como una materia que no se contempla dentro de los aprendizajes significativos del currículo, fue finalmente que en el 2016 donde la enseñanza del Inglés como lengua extranjera se incluye al currículo nacional como materia obligatoria desde segunda educación general básica hasta tercero de bachillerato.

En base a un estudio según (Maridueña-Macancela et al. 2016), dentro de los factores que inciden en el aprendizaje del Inglés existe el incorrecto uso de estrategias didácticas y pedagógicas en el aula, el desconocimiento al uso de los recursos tecnológicos, entre otros. Hay que mencionar además que en la actualidad alrededor de 185 países tuvieron que cerrar obligatoriamente las escuelas a causa del COVID-19 según la (Organización de las Naciones Unidad para la educación la Ciencia y la Cultura [UNESCO], 2020).

Por esta razón el Ministerio de Educación crea el programa aprendamos juntos desde casa, llamado también Plan COVID-19, que permite a los docentes y estudiantes brindar 
la educación desde casa haciendo uso del teletrabajo y educación en línea. Posteriormente las implementaciones de las TIC Tecnologías de la información y la comunicación en las clases han permitido a los docentes aplicar varios modelos pedagógicos y metodologías activas (Ministerio de Educación [MINEDUC], 2020).

Por otro lado, la (Comisión Económica para América Latina y el Caribe [CEPAL], 2020), explica como parte de las medidas adoptadas en la educación por la suspensión de las clases presenciales a causa de la pandemia, ha dado origen a la aplicación de diferentes modalidades de aprendizaje entre ellas la más significativa que es la educación a distancia en tal sentido y con el uso de la tecnología, existen diferentes plataformas que son de ayuda significativa como instrumento en la aplicación de las metodologías educativas.

Como consecuencia hace énfasis en fortalecer las necesidades de los docentes por la exasperación de las competencias digitales. En un reciente estudio realizado por (Baptista-Lucio et al. 2020), se identificó que es necesario plantear un modelo pedagógico a distancia y estar preparado para esta modalidad que será parte del diario vivir de cada uno de los estudiantes hasta las nuevas disposiciones del estado no solo del aspecto tecnológico, sino también de un modelo pedagógico.

Según los resultados del estudio se manifiesta la pertinencia de un verdadero modelo pedagógico como estrategia didáctica llamado aula invertida, en el que la casa se convierte en un espacio para el aprendizaje y los docentes puedan participar en sesiones a distancia. El (Ministerio de educación de Ecuador [MINEDUC], 2018), renueva el programa de enseñanza del idioma Inglés como lengua extranjera [TEFL] como parte del programa de fortalecimiento para que los estudiantes del sistema público mejoren sus conocimientos.

Junto con la implementación de varias actividades haciendo hincapié en enseñanza centrada en el estudiante. Este proyecto con la organización llamada Cuerpo de la paz en el Ecuador permitirá a los docentes fortalecer sus metodologías. En tal sentido la 
aplicación del flipped classroom o dar la vuelta a la clase" en español, es una de las metodologías activas que consiste en direccionar la atención propia del docente y dársela al estudiante para contribuir a su aprendizaje con una serie de elementos.

La importancia de la aplicación de la misma se debe al bajo índice de nivel del idioma ingles en los estudiantes de tercero de bachillerato. En este sentido esta metódica considerada dentro de las metodologías activas le permite al docente aprovechar importantes ventajas sobre todo el desarrollo de la habilidad escucha en el proceso de aprendizaje del idioma Inglés.

Estas permiten que los estudiantes independientemente del enfoque que se lleve a cabo muestren más interés dando como resultado un compromiso por parte de ellos. Dicho con otras palabras, el estudiante se convierte en el actor principal de su propio aprendizaje sobre todo si usamos la técnica del vídeo que se proporciona a la clase teniendo la oportunidad de visualizar los contenidos de la misma las veces necesarias, proporcionando así un mejor entendimiento, y mejorando el desarrollo de la escucha.

Los estudiantes al salir del bachillerato no logran adquirir conocimientos y desarrollar la habilidad de poder escuchar y entender el idioma Inglés totalmente, según la compañía Education First (EF) en una evaluación realizada a 100 países de diferentes regiones en el 2019 Ecuador obtuvo el lugar número 81 obteniendo como resultado un nivel de Inglés "Muy Bajo" con el puntaje de 46.57 que según el Marco Común Europeo [MCER] equivale a un nivel $\mathrm{A} 1$.

El docente cuando distribuye el tiempo de los estudiantes en un aprendizaje individual fuera de la clase, le permite centrarse a la diversidad que existe dentro del salón es decir que se centra en las necesidades específicas del estudiante. La enseñanza del Inglés con la incorporación de las TICs permite desarrollar enfoques para la adquisición del idioma Inglés, el proceso del aprendizaje no simplemente se refiere al dominio de la lengua, sino también su uso apropiado para propósitos comunicativos. 
Este articulo se propone como objetivo general analizar de qué manera se podría desarrollar habilidades de escucha en la asignatura de Inglés mediante el uso de flipped classroom como estrategia didáctica a los estudiantes de tercero de bachillerato de la unidad educativa del Milenio Otto Arosemana Gomez de la ciudad de Milagro provincia del Guayas; identificando los niveles de conocimiento de habilidades de escucha; diiseñando una estrategia didáctica y determinando ventajas y desventajas de su uso.

\section{Referencial teórico}

El uso de las TIC a causa de la pandemia ha traído grandes beneficios en el ámbito educativo de tal forma que para la redacción de esta investigación se toman como referencia artículos científicos y documentos de organismos internacionales dentro de la base de datos de Redalyc y dialnet entre los años 2015-2021. En la búsqueda de cómo la metodología del Flipped Classroom puede desarrollar habilidades en el proceso de adquisición del idioma Inglés, existe una propuesta de innovación educativa creada por (Pérez-Aldeguer et al. 2017), en España, haciendo hincapié en el uso de píldoras audiovisuales en otras palabras lo define también como partes de material didáctico diseñadas específicamente para complementar la clase como lo es el contenido audiovisual facilitando al estudiante la realización de las actividades de manera autónoma a pesar de los resultados positivos menciono también la resistencia en los estudiantes por no asumir sus propias responsabilidades.

La evolución de la tecnología y el acceso a los canales digitales que proporcionan videos se ha convertido en una de las herramientas principales para los docentes lo mencionan (Rajadell \& Garriga-Garzón, 2017), en España, esta metodología de enseñanza se caracteriza por ser mucho más eficiente que cualquier otro recurso basado en textos. También mencionan que el uso de videos como complemento para la clase actúa de manera positiva para los estudiantes en comparación cuando se lo utiliza para remplazar la clase completa. 
Por otro lado, (Perdomo-Rodríguez, 2017), en Colombia, mediante un análisis de aplicación y de los resultados obtenidos, explica que la perspectiva de la metodología no se centra en disminuir el trabajo del docente, sino más bien en crear una exigencia en el estudiante para con la construcción de su conocimiento, en tal sentido es importante indicar que la tecnología no puede remplazar al docente, más bien se convierte en un instrumento para que el docente pueda dar su clase dentro y fuera de ella.

Dentro de la investigación realizada por (Yilorm, 2016), en Chile, menciona principalmente que el idioma en Inglés es uno de los más estudiados como lengua extranjera, dentro del contexto educativo explica que las bases del currículo y el proceso de aprendizaje en el aula no siempre concuerdan haciendo énfasis en que el docente sobre todo del sector público se rige a la traducción del Inglés al idioma natal y la repetición de estructuras gramaticales. En este sentido hace un llamado urgente a definir de manera colaborativa los fundamentos teóricos del proceso de enseñanza y aprendizaje con el fin de terminar con la enseñanza tradicionalista y haciendo el uso de nuevas metodologías que permitan que los estudiantes adquieran los conocimientos y habilidades comunicativas de forma oral y escrita.

En otro orden, (Martínez-Salas, 2019), en Perú, explica como el uso del modelo pedagógico Flipped Classroom beneficia tanto al estudiante en el desarrollo de su propio aprendizaje y al docente en la optimización y mejor uso del tiempo durante las clases. Los estudiantes con mayor dificultad en el aprendizaje del idioma Inglés son aquellos que no cuentan con la ayuda en casa y los conocimientos previos que no adquirieron durante la explicación, por este motivo llegan a clases sin haber realizado sus actividades, en este sentido dentro de la obtención de los resultados detalla que la aplicación y uso de la misma ayuda a los estudiantes a poder desarrollar sus habilidades y esto gracias los cambios y actualizaciones tecnológicas que permiten al docente hacer uso de la misma. Es importante mencionar el aporte de (Marriott-Toledo \& Sànchez-Pèrez, 2017), en Ecuador, los docentes deben considerar que dentro de la metodología del Flipped 
Classroom es importante el uso de la tecnología como instrumento para el desarrollo de la misma, puesto que existen muchos docentes que aún siguen bajo la modalidad tradicional sin considerar las actualizaciones del mundo globalizado. Al hacer uso de esta didáctica se debe considerar 4 aspectos importantes dentro de su desarrollo; contenido o fuente, las tareas enviadas, las tareas realizadas dentro de la clase y las herramientas de evaluación. Siguiendo ese proceso el enfoque tradicional da un giro que permite al docente convertirse en el facilitador del aprendizaje de los estudiantes.

Así mismo, (Reyes-Pérez et al. 2020), en Ecuador, En los casos específicos del fracaso escolar, el uso de la estrategia Flipped Classroom tiene como principal beneficio el trascender la disminución del mismo, según los resultados de la investigación la aplicación de la metodología ayuda de manera significante a la motivación en los estudiantes, mejorando favorablemente el rendimiento académico a su vez participando en actividades colaborativas influyendo en el aprendizaje significativo.

Explican (Carrasco \& Baldivieso, 2016), que las metodologías activas incluidas las del Flipped Learning , modelo al igual que el Flipped Classroom es un estilo de aprendizaje que trabajan juntos de la mano en tal sentido que también adopta el enfoque tradicional para convertirlo en un enfoque participativo. Por tanto, como lo mencionaba las metodologías activas cuentan con el uso de los recursos e información tecnológica brindando así una educación a distancia sin que sea necesariamente así. Es decir que un proceso en donde exista la interacción y motivación junto con el acompañamiento del docente hacia el estudiante.

En este sentido, (Telenchana-Carvajal, 2019), menciona que el aula invertida cambia los procesos tradicionales de la educación, es decir que las actividades se desarrollan fuera del aula por medio de herramientas instrumentos tecnológicos usando videoclips en multimedia, animaciones u otras aplicaciones. Esta metodología se la puede usar en diferentes situaciones ya sea para debate, demostración, o trabajo grupal. 
En tal sentido, (Rea-Hinojoza \& Quinga-Suárez, 2020), en el actual contexto educativo menciona las ventajas del uso de la metodología para con los estudiantes en esta nueva modalidad virtual o también llamada online, a causa del COVID-19 con el fin de prevalecer la salud tanto del docente como el del estudiante y su familia. La educación en tiempos de pandemia ha generado competencias digitales enfocando su atención principalmente a la tecnología brecha que permite al docente tomar como herramienta para la enseñanza y aprendizaje de los estudiantes.

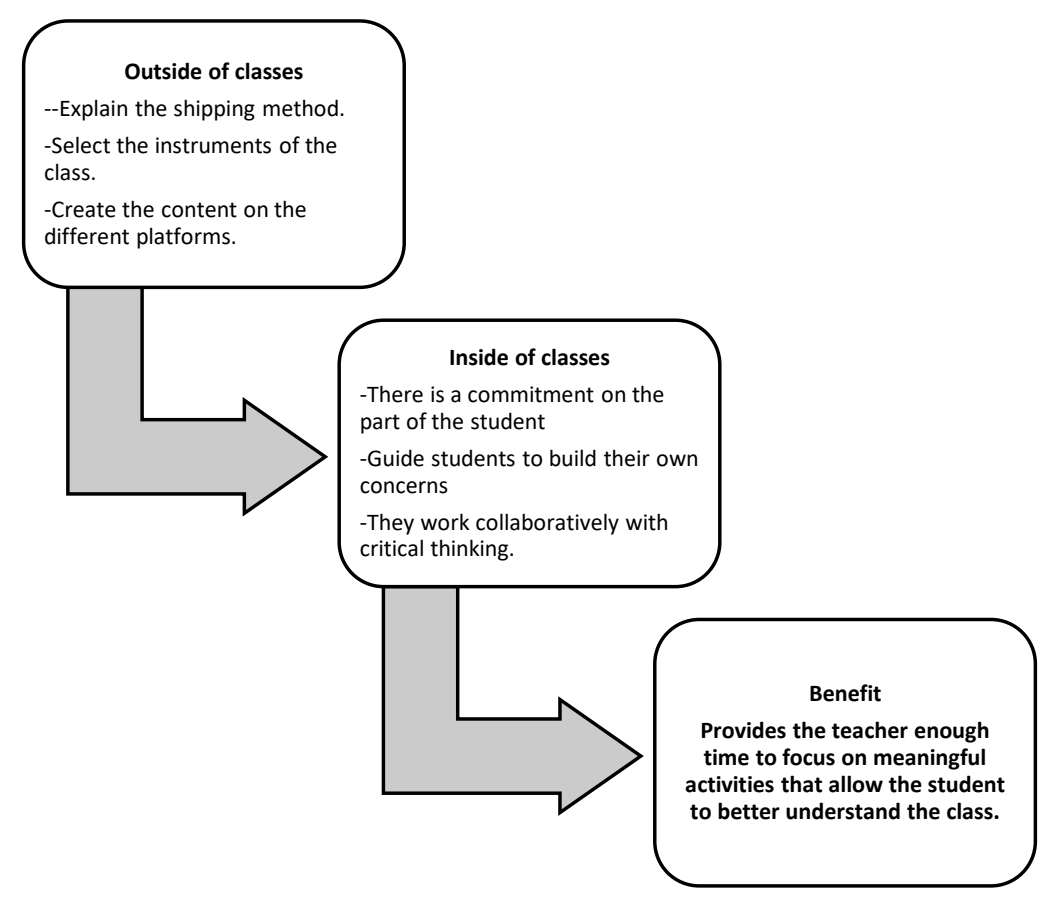

Figura 1. Flipped Classroom Process Elaboración: Los autores.

Dentro de la aplicación del Flipped Classroom, (Alfageme-González \& Nieto-Cano, 2017) hablan sobre las metodologías activas como un grupo de métodos y estrategias acompañados con varias técnicas para el uso de la clase, estas metodologías se basan específicamente en el trabajo cooperativo dándole la oportunidad a los estudiantes de construir su propio conocimiento y pensamiento crítico. 
Es importante mencionar que la actualidad ha exigido al docente adaptarse de una manera abrupta con la tecnología y los nuevos modelos de tecnologías emergentes aplicadas a la formación. Según (Bernate \& Vargas-Guativa, 2020), se refiere aquellas innovaciones tecnológicas que permiten una ventaja muy importante en el uso de las nuevas metodologías, las herramientas que nos brinda las tecnologías emergentes y el uso de los tics dichas herramientas brindan mayor facilidad cuando deben realizar las actividades tanto para el docente como para el estudiante.

\section{METODOLOGÍA}

La presente investigacion tiene un proceso metodologico de carácter no experimental, se plantea un enfoque cuantitativo con cohorte transversal, como lo expresa, (HurtadoTalavera, 2020), el uso adecuado del enfoque ayuda al investigador a conocer que instrumentos son necesarios para la investigación tomando en consideración la situación y el contexto en tal sentido al pasar el tiempo se han ido generando nuevas perspectivas en relación a la búsqueda del conocimiento. El alcance de la investigación es correlacional como lo manifiestan (Hernández et al. 2014), determina la relación, ya sea positiva o negativa de las variables de la investigación, en tal sentido permite conocer las ventajas y desventajas del uso de la metodología flipped classroom con los estudiantes de tercero bachillerato.

Las técnicas de investigación según lo expuesto por (Rojas-Crotte, 2011), son esenciales en el procedimiento de la investigación, permite reconocer que instrumento se aplica, esta se determina a partir del enfoque por esta razón se ha seleccionado la encuesta como instrumento de investigación que a su vez fue validada por el metodo alpha de conbrach con 0,725.

Para poder analizar de qué manera se podría desarrollar habilidades de escucha en la asignatura de Inglés mediante el uso de flipped classroom como estrategia didáctica se ha establecido el tipo de muestreo estratificado siendo la población 166 estudiantes de la 
Unidad Educativa del Milenio Otto Arosemena Gómez del tercero de bachillerato de la jornada matutina y tomando como muestra 118 de ellos divididos en 3 paralelos ( 3 ro bachillerato "A" - 35, 3ro bachillerato "B" -15 estudiantes, 3ro bachillerato "C" -28 estudiantes, 3ro bachillerato "D" -40 estudiantes) en la ciudad de Milagro- Ecuador en tal sentido vale mencionar que al ser docente del aula tuve facilidad de aplicar la metodologia y la recoleccion de la informacion.

\section{RESULTADOS}

El instrumento utilizado para la recopilacion de los datos cualitativos fue una encuesta dirigida a los estudiantes de tercero de bachillerato a traves de un formulario creado en google forms luego de haber aplicado la metodologia del flipped classroom en la asigantura de ingles como parte de la mejora en la habilidad de escucha, los datos fueron analisados con la heramienta SPSS y el análisis de la encuesta fue validada a través de las pruebas de normalidad específicamente la prueba de Kolmogorov por cuanto el número de encuestados superaron las 50 personas, las variables de la encuesta son de carácter parametrico, a continuacion el analisis de cada una de las variables.

\section{Tabla1.}

Acceso a la tecnología y al internet.

\begin{tabular}{clcccc}
\hline & & Frecuencia & Porcentaje & Porcentaje válido & $\begin{array}{c}\text { Porcentaje } \\
\text { acumulado }\end{array}$ \\
\hline \multirow{4}{*}{ Válidos } & Muy Frecuente & 90 & 76,3 & 76,3 & 76,3 \\
& Frecuente & 23 & 19,5 & 19,5 & 95,8 \\
& A veces & 5 & 4,2 & 4,2 & 100,0 \\
\cline { 2 - 6 } & Total & 118 & 100,0 & 100,0 & \\
\hline
\end{tabular}

Fuente: Encuesta 
En la tabla 1 se observa que el $95.8 \%$ de los estudiantes tienen acceso frecuente con la tecnologia y el internet, lo cual se evidencia su alcance para los elementos antes mencionados, dando viabilidad para el uso del flipped classroom en la asignatura de ingles.

\section{Tabla 2}

Uso de plataformas digitales.

\begin{tabular}{clcccc}
\hline & & Frecuencia & Porcentaje & Porcentaje válido & $\begin{array}{c}\text { Porcentaje } \\
\text { acumulado }\end{array}$ \\
\hline \multirow{4}{*}{ Válidos } & Muy Frecuente & 70 & 59,3 & 59,3 & 59,3 \\
& Frecuente & 44 & 37,3 & 37,3 & 96,6 \\
& A veces & 4 & 3,4 & 3,4 & 100,0 \\
\cline { 2 - 6 } & \multicolumn{1}{c}{ Total } & 118 & 100,0 & 100,0 & \\
\hline
\end{tabular}

Fuente: Encuesta

En la tabla 2 se observa que el $96.6 \%$ de los estudiantes usan las plataformas digitales con frecuencia, estos datos aceveran una cantidad considerable de estudiantes, dando luz al uso de las mismas para la aplicacion de la metodologia flipped classroom en la asignatura de ingles.

\section{Tabla 3.}

Uso de tecnología factible.

\begin{tabular}{llcccc}
\hline & Frecuencia & Porcentaje & $\begin{array}{c}\text { Porcentaje } \\
\text { válido }\end{array}$ & $\begin{array}{c}\text { Porcentaje } \\
\text { acumulado }\end{array}$ \\
\hline \multirow{4}{*}{ Válidos } & Totalmente de acuerdo & 70 & 59,3 & 59,3 & 59,3 \\
& De acuerdo & 44 & 37,3 & 37,3 & 96,6 \\
& A veces & 4 & 3,4 & 3,4 & 100,0 \\
\cline { 2 - 6 } & Total & 118 & 100,0 & 100,0 & \\
\hline
\end{tabular}

Fuente: Encuesta 
En la tabla 3 se observa que el $96.6 \%$ de los estudiantes usan la tecnologia como via factible para poder recibir las clases, tomando como contexto actual las clases en linea, los datos mostrados reflejan la concordancia y la viabilidad de la tecnologia como herramienta principal al uso de la metodologia flipped classroom en la asignatura de ingles.

\section{Tabla 4.}

Uso de las aplicaciones Classroom y Mentimeter.

\begin{tabular}{llllll}
\hline & & & Porcentaje & Porcentaje \\
& & Frecuencia & Porcentaje & válido & acumulado \\
\hline \multirow{2}{*}{ Válidos } & Totalmente de acuerdo & 67 & 56,8 & 56,8 & 56,8 \\
& De acuerdo & 43 & 36,4 & 36,4 & 93,2 \\
& A veces & 8 & 6,8 & 6,8 & 100,0 \\
\cline { 2 - 6 } & Total & 118 & 100,0 & 100,0 & \\
\hline
\end{tabular}

Fuente: Encuesta.

En la tabla 4 se observa que el $93.2 \%$ de los estudiantes conocian el uso de las aplicaciones classroom y mentimeter dando como resultado ventajoso tanto para el estudiante como para el docente de tal manera que estos instrumentos beneficien el uso de la metodologia flipped classroom en la asignatura de ingles en la optimizacion de tiempo. 
Tabla 5.

Uso de nuevos programas digitales.

\begin{tabular}{llcccc}
\hline & Frecuencia & Porcentaje & $\begin{array}{c}\text { Porcentaje } \\
\text { válido }\end{array}$ & $\begin{array}{c}\text { Porcentaje } \\
\text { acumulado }\end{array}$ \\
\hline \multirow{4}{*}{ Válidos } & Totalmente de acuerdo & 75 & 63,6 & 63,6 & 63,6 \\
& De acuerdo & 36 & 30,5 & 30,5 & 94,1 \\
& A veces & 7 & 5,9 & 5,9 & 100,0 \\
\cline { 2 - 6 } & Total & 118 & 100,0 & 100,0 & \\
\hline
\end{tabular}

Fuente: Encuesta.

En la tabla 5 se observa que el $94.1 \%$ que los estudiantes habian tenido una experiencia similar con los programas digitales antes en la clase de ingles, en tal sentido resulta ventajoso tanto para el estudiante como para el docente como parte de las herramientas que beneficien el uso de la metodologia flipped classroom en la asignatura de ingles para la optimizacion de tiempo. 
Tabla 6.

Asociación del acceso a la tecnología al internet y el uso de plataformas digitales.

\begin{tabular}{|c|c|c|c|c|c|}
\hline \multicolumn{6}{|l|}{ Pruebas de chi-cuadrado } \\
\hline & & Valor & gl & \multicolumn{2}{|c|}{ Sig. asintótica (bilateral) } \\
\hline \multicolumn{2}{|c|}{ Chi-cuadrado de Pearson } & $22,626^{a}$ & 4 & \multicolumn{2}{|c|}{, 000} \\
\hline \multicolumn{2}{|c|}{ Razón de verosimilitudes } & 10,009 & 4 & \multicolumn{2}{|c|}{,040 } \\
\hline \multicolumn{2}{|c|}{ Asociación lineal por lineal } & 5,196 & 1 & \multicolumn{2}{|c|}{,023 } \\
\hline \multicolumn{2}{|c|}{$\mathrm{N}$ de casos válidos } & 118 & & & \\
\hline & & \multicolumn{3}{|c|}{ Uso de plataformas digitales } & \multirow{2}{*}{ Total } \\
\hline & & Muy Frecuente & Frecuente & A veces & \\
\hline \multirow{3}{*}{$\begin{array}{c}\text { Acceso a la tecnología y al } \\
\text { internet }\end{array}$} & Muy Frecuente & 55 & 34 & 1 & 90 \\
\hline & Frecuente & 14 & 8 & 1 & 23 \\
\hline & A veces & 1 & 2 & 2 & 5 \\
\hline \multicolumn{2}{|l|}{ Total } & 70 & 44 & 4 & 118 \\
\hline
\end{tabular}

Fuente: Encuesta.

Como se observa en la Tabla 6 , el valor del chi-cuadrado muestra un valor de 0,000 menor a 0,05 y manifiesta una correlación con los estudiantes que tienen acceso a la tecnología y el uso de las plataformas digitales para las clases. Esta aseveración, claramente nos demuestra que podemos aplicar la metodología Flipped con los estudiantes para mejorar la habilidad escucha en la asignatura de Inglés.

\section{PROPUESTA}

De acuerdo al análisis de los resultados obtenidos en la investigacion y considerando la educacion virtual en el contexto actual a causa de la pandemia por COVID-19, es factible la recomendación a los docentes de la Unidad Educativa del Milenio Otto Arosemena Gomez que sea posible el uso de la metodologia flipped classroom en la asignatura de ingles para mejorar la habilidad de escucha a traves de videos grabados y haciendo uso 
de programas y herramientas digitales, de tal manera que se optimice el tiempo durante la clase y facilite la repeticion de los videos a los estudiantes para poder realizar las actividades asignadas. Como se muestra en la Figura 1, los procesos como medidas para la aplicación en la la unidad educativa se propone lo siguiente.

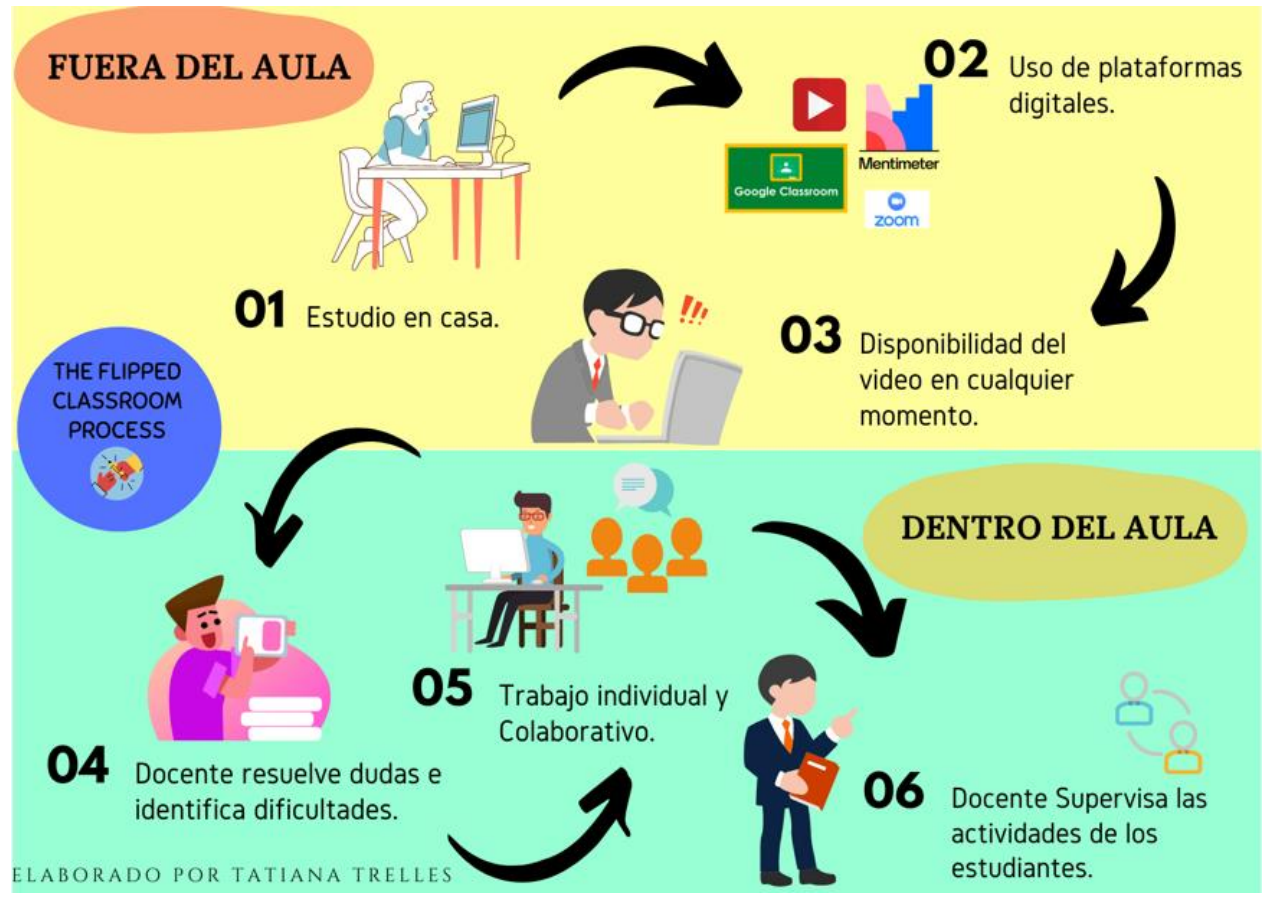

Figura 1. Aplicación del Flipped Classroom en la dsignatura de Ingles Elaboración: Los autores.

Los detalles para el proceso de la aplicación de la metodologia de flipped classroom en la asignatura de Ingles se demuestran a continucion: 


\section{Primer momento fuera de clases:}

-Docente debera preparar material didactico relacionado con el tema de la clase que se presentara en dia posterior, puede ser fuente propia o recursos digitales reutilizables.

-Docente debera subir el video o facilitar viabilidad para que pueda ser visto por el estudiante a traves de plataformas recomendadas como youtube y classroom.

-Estudiante debera revisar la asignacion y repetir la veces necesarias para poder entender de que se trata el tema.

-Estudiante debera revisar las herramientas facilitadas por el docente y verificar las asignaciones.

\section{Segundo momento durante las clases:}

-El docente guiara la actividad a realizar dentro la clase planificada.

-El docente facilitara los temas y recursos a los estudiantes.

-El estudiante explicara en sus propias palabras que fue lo que entendio para que el docente valide la informacion o a su vez reforzar.

\section{Ventajas del uso de flipped clasrrom en la clase de ingles}

-Estudiante se convierte en el protagonista de su aprendizaje y el docente en un guia.

-El uso de las tecnologias motiva al estududiante haciendo uso de las diferentes herramientas digitales que les permite tomar decisiones, desarrollar conocimientos y habilidades y asumir responsabilidades.

-Docente invierte y optimiza su tiempo en la clase lo que contribuye al estudiante a un aprendizaje mas significativo que le sea de utilidad para el futuro. 
- Los estudiantes pueden pausar y reproducir el video de la clase cuantas veces sean necesarias lo que les permite comprender correctamente y a resolver sus inquietudes. -Mayor facilidad para poder entregar las tareas a traves de las heramientas digitales y de la misma forma quien revisa la actividad en este caso el docente.

\section{CONCLUSIONES}

El Flipped Classroom o también conocido como aula Invertida es un modelo de enseñanza que forma parte de las metodologías activas, es decir que es una enseñanza basada en el aprendizaje del estudiante que permite su uso en la modalidad online o semipresencial.

Tomando de referencia que a causa de la pandemia los estudiantes tuvieron que recibir sus clases desde casa de manera online, con este estudio se evidenció la posibilidad de poder aplicar el Flipped Classroom para la asignatura de Inglés, buscando la mejora en la habilidad escucha con el uso de videos y plataformas virtuales que le permitan al estudiante adaptarse al ritmo y necesidades que posea, de esta forma el docente tendrá la atención más personalizada para cada uno de los estudiantes dentro de su clase.

El aprendizaje Invertido como estrategia didáctica para el desarrollo de habilidades de escucha en la asignatura de Inglés es una gran opción para ser puesta en manifiesto en el aula de clases, puesto que permite la participación activa de los estudiantes no solo en la destreza de escuchar, sino en las de hablar y escribir.

\section{FINANCIAMIENTO}

No monetario.

\section{AGRADECIMIENTOS}

A la Universidad Católica de Cuenca; por impulsar el desarrollo de este proceso investigativo desde la praxis educativa. 


\section{REFERENCIAS CONSULTADAS}

Alfageme-González, M. \& Nieto-Cano, J. M. (2017). Los docentes de la enseñanza obligatoria en España y las actividades de formación continua. [Teachers of compulsory education in Spain and continuous training activities]. Perfiles Educativos, XXXIX(158), 148-165. https://n9.cl/z7u07

Baptista-Lucio, P., Almazán Zimerman, A., \& Loeza Altamirano, C. A. (2020). Encuesta Nacional a Docentes ante el COVID-19. Retos para la educación a distancia. Revista Latinoamericana de Estudios Educativos, 50(ESPECIAL), 41-88. https://n9.cl/o7v4f

Carrasco, S., \& Baldivieso, S. (2016). Educación a distancia sin distancias [Distance education without distances]. Universidades, 70, 7-26. https://www.redalyc.org/pdf/373/37348529003.pdf

Reyes-Pérez, K., García-Herrera, D., Mena-Clerque, S., \& Erazo-Álvarez, J. (2020). Flipped Classroom como estrategia metodológica en la Carrera de Imagenología: propuesta de aplicación de Storyflipped [Flipped Classroom as a methodological strategy in the Imaging Career: application proposal of Storyflipped]. EPISTEME KOINONIA, 3(1), 231-254. http://dx.doi.org/10.35381/e.k.v3i1.1005

Comisión Económica para América Latina y el Caribe [CEPAL]. (2020). La educación en tiempos de la pandemia de COVID-19 [COVID-19]. https://n9.cl/mgw0

Hernández-Sampieri, R., Fernández-Collado, C., \& Baptista-Lucio, M. (2014). Metodología de la Investigación [Investigation Methodology] (Sexta). McGRAWHILL.

Hernández-Hernández, M, \& Izquierdo-Sandoval, M. (2020). Cambios curriculares y enseñanza del inglés. Cuestionario de percepción docente [Curricular changes and English language teaching: Validation of a teacher perception questionnaire]. Sinéctica, (54), e1042. Epub 07 de agosto de 2020. https://doi.org/10.31391/s2007-7033(2020)0054-012

Hurtado-Talavera, F. J. (2020). Fundamentos Metodológicos de la Investigación: El Génesis del Nuevo Conocimiento. Revista Scientific, 5(16), 99-119. https://www.redalyc.org/journal/5636/563662985006/html/ 
Maridueña-Macancela, J., Ledesma Acosta, B., \& Macias Arroyo, F. (2016). Módulos de Inglés y su repercusión en las destrezas del idioma [English modules and their impact on language skills]. CIENCIA UNEMI, 9(19), 68-75. https://www.redalyc.org/journal/5826/582661268008/582661268008.pdf

Marriott-Toledo, H. M., \& Sànchez-Pèrez, L. M. (2017). Flipping the Classroom: Developing Teaching Skills for Future In-Service English Teachers. INNOVA Research Journal, 2(8), 119-129. https://doi.org/10.33890/innova.v2.n8.2017.345

Bernate, J. A., \& Vargas-Guativa, J. A. (2020). Desafíos y tendencias del siglo XXI en la educación superior [Challenges and trends of the 21 st century in higher education]. Revista De Ciencias Sociales, 26, 141-154. https://doi.org/10.31876/rcs.v26i0.34119

Martínez-Salas, M. M. (2019). El modelo pedagógico de clase invertida para mejorar el aprendizaje del idioma Inglés. Investigación Valdizana, 13(4), 204213. https://doi.org/10.33554/riv.13.4.486

Ministerio de Educación [MINEDUC]. (2014). Ministro de Educación explica el fortalecimiento del aprendizaje del Inglés en el sistema educativo del país. https://n9.cl/6d2h

Ministerio de Educación [MINEDUC]. (2018). Educación y representantes del Cuerpo de Paz se reúnen para fortalecer la enseñanza del idioma Inglés [Education and representatives of the Peace Corps meet to strengthen the teaching of the English language]. https://n9.cl/p9dh3

Ministerio de Educación [MINEDUC]. (2020). Plan Educativo COVID 19 [COVID Education Plan 19]. https://educacion.gob.ec/plan-educativo-covid-19/

Organización de las Naciones Unidad para la educación la Ciencia y la Cultura [UNESCO]. (2020). 290 millions of estudiantes sin clases por el COVID-19: a UNESCO report on the world [290 millions of estudiantes sin clases por el COVID19: a UNESCO report on the world]. https://n9.cl/arct

Perdomo-Rodríguez William. (2017). Ideas y reflexiones para comprender la metodología Flipped Classroom. [Ideas and reflections to understand the Flipped Classroom methodology]. Revista Virtual Universidad Católica Del Norte, O(50), 143-161. https://n9.cl/xjswb 
Pérez-Aldeguer, S., Castellano-Pérez, G., \& Pina, A. (2017). Propuestas De Innovación Educativa En La Sociedad De La Información [Proposals for Educational Innovation in the Information Society]. Adaya Press, 136-146. https://n9.cl/uvo48

Rajadell, M., \& Garriga-Garzón, F. (2017). Educational videos: After the why, the how. Intangible Capital, 13(5), 902-922. https://doi.org/10.3926/ic.1042

Rea-Hinojoza, Á. A., \& Quinga-Suárez, C. (2020). Educación en tiempos de emergencia , una educación en transición [Education in times of emergency, an education in transition]. Killkana Sociales, 4(2), 43-50. https://n9.cl/295gd

Rojas-Crotte, I. R. (2011). Elementos para el diseño de Técnicas de Investigación: Una propuesta de definiciones y procedimientos en la investigación científica [Elements for the design of Research Techniques: A proposal for definitions and procedures in scientific research]. Tiempo de Educar, 12(24), 277-297. https://www.redalyc.org/pdf/311/31121089006.pdf

Telenchana-Carvajal, S. P. (2019). The flipped classroom method in english speaking skill development (Tesis de maestría). Universidad Técnica de Ambato. Dirección de Posgrado. Maestria En La Enseñanza Del Idioma Inglés Como Lengua Extranjera, 153. https://n9.cl/a83kv

Yilorm, Y. (2016). Proceso de enseñanza aprendizaje de la lengua inglesa en escuelas públicas chilenas: ¿Producción o reproducción? [The learning teaching process of English as a foreign language in Chilean Public Schools: Production or reproduction?]. Estudios pedagógicos (Valdivia), 42(especial), 103116. https://dx.doi.org/10.4067/S0718-07052016000300009 